\title{
Cardiotoxicidad inducida por la quimioterapia desde las bases moleculares hasta la perspectiva clínica
}

\author{
Camilo A. Velásquez ${ }^{\mathrm{a}, *}$, Miguel González ${ }^{\mathrm{b}}$, Marie C. Berrouet ${ }^{\mathrm{c}}$ y Nicolás Jaramillo ${ }^{\mathrm{d}}$ \\ a Universidad CES. Cardiovital Investigaciones - CEMDE-Universidad CES. Medellín, Colombia \\ b Departamento de Medicina Interna, Rutgers University - New Jersey Medical School. Newark, New Jersey, Estados Unidos \\ c Hospital General de Medellín/Clínica SOMA-CEMDE. Universidad CES. Medellín, Colombia \\ d Clínica Las Américas - CEMDE-Fundación Ciencia Vital-Cardiovital Investigaciones. Medellín, Colombia
}

Recibido el 26 de agosto de 2015; aceptado el 1 de octubre de 2015

Disponible en Internet el 21 de noviembre de 2015

\section{PALABRAS CLAVE \\ Corazón; \\ Complicaciones; \\ Insuficiencia \\ cardiaca; \\ Tratamiento; \\ Radicales libres}

\section{KEYWORDS}

Heart;

Complications;

Cardiac failure;

Treatment;

Free radicals

\begin{abstract}
Resumen En la actualidad, el cáncer representa una epidemia que puede ser considerada como un problema de salud pública. Es por esto que el desarrollo farmacológico ha tomado un auge en pro de mejorar las tasas de sobrevida y remisión. No obstante, esto ha sido oscurecido por la toxicidad de estas terapias. La cardiotoxicidad es un efecto adverso conocido de la terapia oncológica, que limita en muchas ocasiones una adecuada administración de regímenes con disminución de la eficacia; por esto, el entendimiento de las bases fisiopatológicas y moleculares son esenciales para determinar medidas preventivas y terapéuticas que permitan una disminución en la incidencia de la toxicidad, sin influir en la efectividad de la terapia oncológica.

A continuación se realiza una revisión de los efectos adversos de la quimioterapia en el sistema cardiaco, incluyendo desde sus bases moleculares hasta sus implicaciones clínicas.

(C) 2015 Sociedad Colombiana de Cardiología y Cirugía Cardiovascular. Publicado por Elsevier España, S.L.U. Este es un artículo Open Access bajo la licencia CC BY-NC-ND (http://creativecommons.org/licenses/by-nc-nd/4.0/).
\end{abstract}

\section{Chemotherapy-induced cardiotoxicity from molecular bases to clinical perspective}

Abstract Nowadays cancer represents an epidemic that can be considered a public health problem. That is why pharmacological development has emerged for improving survival and remission rates. Nevertheless, this has been obscured by the toxicity of these therapies. Cardiotoxicity is a known adverse event of oncologic therapy that many times limits a correct regimen of administration thus lowering efficacy; therefore, understanding pathophysiological

\footnotetext{
* Autor para correspondencia.

Correos electrónicos: c_velasquezm@outlook.com, camima1792@gmail.com (C.A. Velásquez).
} 
and molecular bases is essential in order to determine preventive and therapeutical measures that can allow a reduction in the incidence of toxicity, without interfering in the efficacy of the cancer therapy.

We hereby describe a review of the adverse effects of chemotherapy in the cardiac system, from molecular bases to clinical implications.

(c) 2015 Sociedad Colombiana de Cardiología y Cirugía Cardiovascular. Published by Elsevier España, S.L.U. This is an open access article under the CC BY-NC-ND license (http://creativecommons.org/licenses/by-nc-nd/4.0/).

\section{Introducción}

El cáncer es un problema de salud pública de gran envergadura en el mundo. Es la segunda causa de mortalidad en los Estados Unidos y se espera que aproximadamente entre los años 2025-2030, sobrepase a las enfermedades cardiovasculares como la principal causa de muerte. Durante el año 2015, en Estados Unidos se han presentado aproximadamente 165.830 nuevos casos de cáncer, lo que equivale a más de 4.500 casos diagnosticados cada día; y se estima que 589.430 americanos morirán en el año 2015 secundario a una afección oncológica representando 1.600 muertes diarias ${ }^{1}$. Aunque la tasa de incidencia ajustada a la edad ha disminuido en los últimos 10 años, el número total de pacientes que padecen de cáncer continúa creciendo; esto se asocia a una población de mayor edad y a una mayor sobrevida gracias a la detección temprana y los avances tecnológicos en el tratamiento ${ }^{2}$.

En los últimos 50 años, la quimioterapia (QT) y la radioterapia (RT) se han convertido en dos de los pilares del tratamiento de varios tipos de cáncer. Su uso ha permitido un aumento en la sobrevida de los pacientes con cáncer; no obstante, la frecuencia de su uso para lograr remisión, las dosis y su mecanismo de acción pueden generar efectos secundarios en los pacientes. Uno de los efectos secundarios más preocupantes generados por la QT y la RT es la cardiotoxicidad. A pesar de tener muchas manifestaciones, una de las principales son los síntomas de la falla cardiaca, secundarios a un daño a nivel del miocardio por la toxicidad directa e indirecta de las terapias antineoplásicas ${ }^{3,4}$. Debido a esto, la función cardiaca es una de las variables de dosis limitantes durante el tratamiento oncológico, ya que contribuye a la morbilidad y la mortalidad de la población expuesta.

La evaluación cardiovascular de los pacientes expuestos a QT, el análisis del riesgo, la prevención y la mitigación de la injuria cardiaca, el monitoreo de la función cardiaca durante y después de la terapia, el avance en los medicamentos utilizados, la prevención y el tratamiento de la cardiotoxicidad, son medidas que se deben tomar de forma interdisciplinaria para lograr un enfoque global del paciente y facilitar una evolución adecuada; es por esto que la cardio-oncología surge como una disciplina necesaria para el enfoque y tratamiento integral de estos pacientes.

\section{Definición de cardiotoxicidad}

La cardiotoxicidad es un término general usado para describir una toxicidad que afecta al corazón de forma directa o indirecta; de forma directa mediante un daño a la estructura del corazón e indirecta a través de los estados trombogénicos y las alteraciones hemodinámicas del flujo sanguíneo ${ }^{5}$. A pesar del auge en la investigación en este campo, hasta el momento no se ha logrado encontrar una definición clara de esta entidad. Para solucionar la ausencia de una definición, el comité de evaluación y revisión cardiaca (Cardiac Review and Evaluation Committee) ha definido la cardiotoxicidad como la presencia de una o más de las siguientes condiciones en pacientes que han recibido tratamientos oncológicos ${ }^{6}$ :

- La cardiomiopatía caracterizada por la disminución en la función ventricular izquierda (FVI) que sea global o más severa hacia el septum.

- Los síntomas de la falla cardiaca.

- Los signos asociados con la falla cardiaca, incluyendo la presencia de S3, taquicardia o ambos.

- La disminución de al menos el $5 \%$ de la fracción de eyección (FE) con valores menores al $55 \%$ con signos o síntomas de la falla cardiaca, o una disminución de al menos el 10\% en la FE con valores menores del $55 \%$ sin la presencia de signos o síntomas ${ }^{6}$.

La cardiotoxicidad se puede presentar de forma temprana durante el tratamiento, hasta 40 años después de finalizada la terapia ${ }^{5,7}$. Entendiendo la variable tiempo, el daño miocárdico se clasifica en:

1. Agudo o subagudo: cuando se desarrolla desde el inicio del tratamiento hasta dos semanas después de terminado.

2. Crónico: cuando la toxicidad aparece posterior a un año de completar la terapia, a su vez la cardiotoxicidad crónica se divide en dos estados ${ }^{8,9}$ : durante el primer año posterior a la terapia se denominan temprana y las que ocurren años después a la finalización de la terapia se denominan tardías ${ }^{10,11}$.

\section{Cardiotoxicidad inducida por la quimioterapia}

El objetivo en la quimioterapia es la inhibición de la división celular; para lograr esto se debe recurrir a diversos grupos de medicamentos citotóxicos, agentes hormonales, inhibidores de la proteina kinasa y los anticuerpos monoclonales (fig. 1), sin embargo, su efecto clínico no es selectivo, afectando las células no cancerígenas propias del organismo y generando toxicidad $^{12}$. 


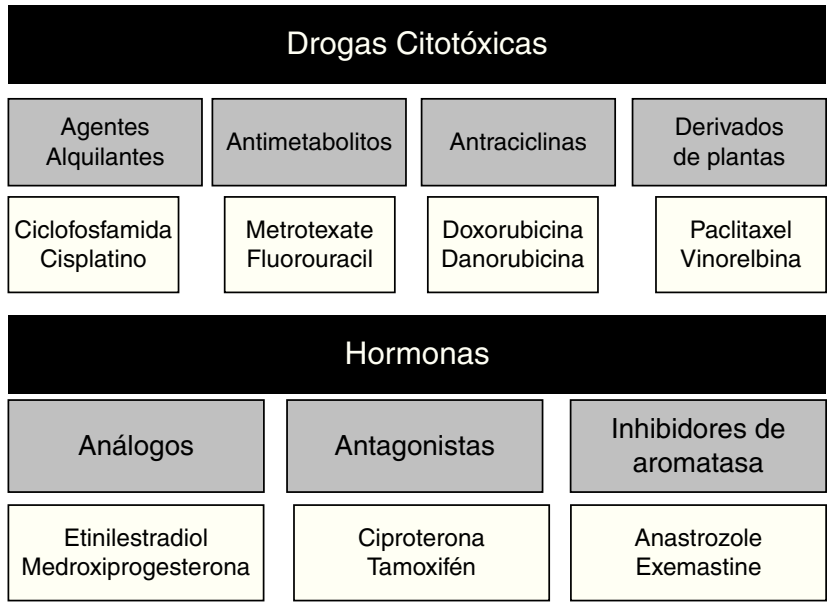

Inhibidores de protein quinasa

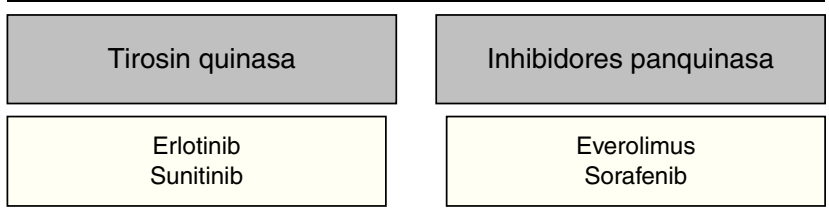

Anticuerpos monoclonales

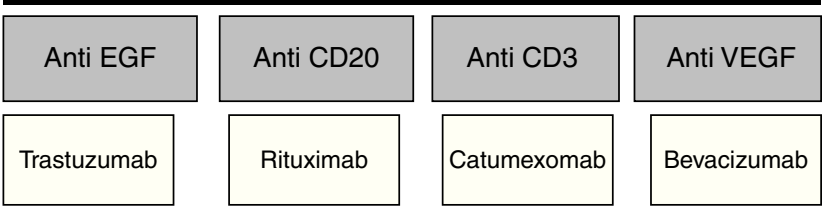

Figura 1 Clasificación de agentes antineoplásicos y representantes de cada clase ${ }^{12}$.

Los efectos tóxicos de los agentes quimioterapéuticos sobre el sistema cardiovascular son diversos, siendo el más común la falla cardiaca con disfunción sistólica ventricular. A su vez, la toxicidad se puede manifestar como: la hipertensión arterial, la enfermedad tromboembólica, el compromiso pericárdico, las arritmias y la isquemia miocárdica. Estas manifestaciones se presentan cuando hay alteraciones en: la estabilidad de la membrana y la función contráctil o al generarse disfunción de las organelas y el estrés oxidativo. Alteraciones en la homeostasis del calcio, la oxidación mitocondrial, la síntesis de proteínas contráctiles, la expresión de genes, la apoptosis, la activación neurohormonal y la generación de especies libres de oxígeno, son los principales mecanismos responsables del daño miocárdico ${ }^{13}$.

Dentro de los agentes antineoplásicos, los medicamentos que tienen el riesgo de producir la cardiotoxicidad se clasifican en dos tipos:

1. Tipo ı: la cardiotoxicidad con mecanismo similar a las antraciclinas. Su toxicidad cardiaca es dosis dependiente y es productora de un daño cardiaco irreversible.

2. Tipo ॥: la cardiotoxicidad con mecanismo similar al trastuzumab, relacionado con un daño cardiaco reversible que permite una recuperación de la funcionalidad y un reinicio del régimen si está indicado. Esto se logra, debido a que no hay cambios ultraestructurales en los miocitos $^{14,15}$.

Anteriormente, la principal asociación entre la cardiotoxicidad y los quimioterapéuticos fue con las antraciclinas, específicamente con dosis acumuladas $>550 \mathrm{mg} / \mathrm{m}^{2}$. La administración repetida de estos medicamentos se asociaba a daño celular e intersticial permanente ${ }^{16}$. Con los nuevos medicamentos, también se amplió el espectro de los cardiotóxicos. Ciertos medicamentos que actúan sobre los receptores de tirosin kinasa o el receptor 2 del factor de crecimiento epidérmico Her2/neu presentan efectos clínicos no deseados sobre el sistema cardiovascular que a diferencia de las antraciclinas no es asociada al uso de dosis acumuladas $^{6,17}$.

\section{Mecanismos fisiopatológicos generales y específicos en los medicamentos}

Generalmente, la cardiotoxicidad inducida por QT se debe a mecanismos multifactoriales entre los que se encuentran:

1. La producción de radicales libres (ROS).

2. Los defectos en la estructura y función mitocondrial.

3. La alteración en la homeostasis del calcio y el hierro.

4. La alteración en la expresión genética ${ }^{18}$.

La consecuencia final es la muerte miocárdica celular, demostrada por la inducción de apoptosis ${ }^{18}$, asociada a la deprivación del crecimiento y supresión de la angiogénesis, comprometiéndose la capacidad de reparación ${ }^{19}$, (fig. 2).

\section{Agentes Tipo I «efecto antraciclina»}

Ese tipo de medicamentos son empleados en el tratamiento de las neoplasias como: el cáncer de mama, los sarcomas, las leucemias y los linfomas ${ }^{14}$. Su presentación puede ser aguda o crónica, el efecto agudo es dosis independiente y transitorio relacionado con la hipersensibilidad tipo 1, mientras que su efecto crónico es dependiente de la dosis acumulativa ${ }^{20}$. Entre los factores de riesgo para la cardiotoxicidad se encuentran: el género femenino, la administración en bolos intravenoso, el uso de dosis acumuladas, la enfermedad cardiovascular previa, las alteraciones electrolíticas, la radiación mediastinal y las alteraciones genéticas. Su mecanismo se relaciona con el daño producido por los radicales libres en el cual la reducción del grupo quinona en el anillo $B$ de los antracíclicos lleva a la formación de un radical semiquinona el cual se oxida y genera radicales libres como el superóxido con la formación del peróxido de hidrógeno el cual interactúa con el miocardio produciendo un ambiente de desequilibrio entre los mecanismos antioxidantes y las sustancias proinflamatorias, predisponiendo al daño por la reducción del glutatión peroxidasa que hace parte de sus mecanismos antioxidantes y se afecta con el uso de estos medicamentos. La formación de un complejo de hierro férrico con la doxorrubicina cataliza esta reacción, aumentando los ROS, los cuales contribuyen a la conversión de hierro ferroso en férrico, destruyendo las membranas celulares y el retículo endoplasmático, lo que produce un descenso en el calcio intracelular y una disminución en la 

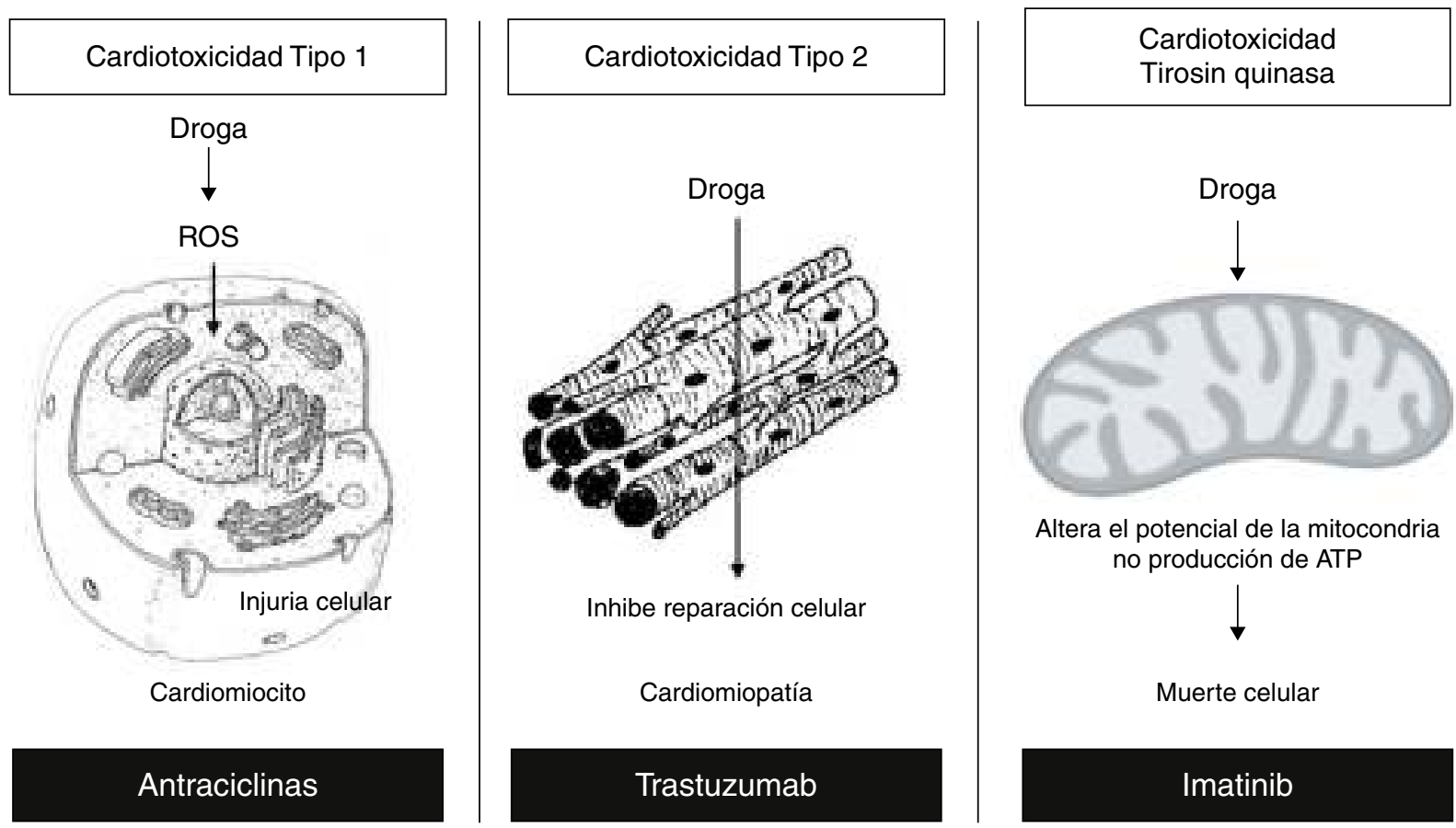

Altera el potencial de la mitocondria no producción de ATP

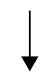

Muerte celular

Figura 2 Mecanismos relacionados con los tipos de antineoplásicos. Reproducida y modificada con permiso del autor ${ }^{21}$. EGF: factor de crecimiento epidérmico. VEGF: factor de crecimiento vascular endotelial.

contractibilidad. A su vez, las citoquinas inflamatorias inducen la liberación de histamina, factor de la necrosis tumoral alfa y la interleuquina 2 , estas citoquinas inducen la cardiomiopatía dilatada y la disfunción betaadrenérgica. A parte del estrés oxidativo, las topoisomerasas también se han implicado en la toxicidad asociada a las antraciclinas, la actividad antitumoral de la doxorrubicina se explica por la formación de un complejo ternario con una de las isoenzimas conocida como Top2a-doxorrubicina-ADN. Estos cambios se han asociado a un aumento en la apoptosis ${ }^{21,22}$.

El efecto tóxico de las antraciclinas se manifiesta dependiendo del agente involucrado y su dosis respectiva: la doxorrubicina (> $500 \mathrm{mg} / \mathrm{m}^{2}$ ), la daunorrubicina $\left(>450 \mathrm{mg} / \mathrm{m}^{2}\right)$, la epirrubicina $\left(>720 \mathrm{mg} / \mathrm{m}^{2}\right)$, la idarrubicina $\left(>90 \mathrm{mg} / \mathrm{m}^{2}\right)$ y la mitoxantrona $\left(>120 \mathrm{mg} / \mathrm{m}^{2}\right)^{23}$. La valrrubicina y la epirrubicina son dos medicamentos que han demostrado disminución en la cardiotoxicidad por su efecto local y su biotransformación en el hígado, respectivamente ${ }^{18,24}$.

\section{Agentes Tipo ॥: «efecto trastuzumab»}

Uno de los principales agentes biológicos asociados a la cardiotoxicidad es el trastuzumab, utilizado en el tratamiento del cáncer de mama; el cual actúa uniéndose al dominio extracelular del HER2 internalizándolo. Este factor de crecimiento epidérmico es un receptor tirosin quinasa transmembrana que actúa como proto-oncogen y se relaciona con la regulación del crecimiento celular, se sobreexpresa en el $25 \%$ de los cánceres de mama y se asocia con el mal pronóstico; en el corazón se asocia con la neuregulina (ligando peptídico de HER3 y HER4), que al unirse con HER4 permite una heterodimerización con HER2 con subsecuente fosforilación y activación de varias vías de señalización que aumentan: el contacto celular, el acoplamiento mecánico y promueven la sobrevida y la función contráctil, los cuales son necesarios para el desarrollo y la supervivencia de los miocitos $\operatorname{cardiacos}^{25}$. Entre los factores de riesgo para la toxicidad por este grupo de medicamentos se encuentran: la exposición previa a las antraciclinas, la edad mayor a 50 años y la clasificación de funcionalidad de Nueva York (NYHA) mayor o igual a $2^{26}$. La exposición al trastuzumab lleva a un efecto antiproliferativo en aquellas células que los expresan, entre ellas encontramos los miocitos cardiacos los cuales al expresar este receptor previenen la apoptosis, y una vez inhibido, aceleran la apoptosis y disminuyen la recuperación funcional llevando a una disfunción cardiaca y posteriormente a la falla cardiaca ${ }^{27}$, (fig. 3).

La terapia combinada con agentes de tipo I y ॥ se asocian con una incidencia mayor de la cardiotoxicidad; esta incidencia es del $27 \%$ comparada con un $3-7 \%$ cuando se utilizan agentes individuales como el trastuzumab, representando una limitante a la hora de elegir la terapia antineoplásica ${ }^{21}$, especialmente, en aquellos pacientes con: edad avanzada (> 80 años), enfermedad coronaria e hipertensión; en donde el trastuzumab se ha relacionado con mayor incidencia de la falla cardiaca ${ }^{28}$.

La cardiotoxicidad producida por medicamentos como: los inhibidores de Tirosin quinasa (imatinib, dasatinib, nilotinib), se ha asociado a disfunción mitocondrial. Las células cardiacas presentan altos requerimientos de ATP, por lo cual son susceptibles a la disminución en su concentración, estos medicamentos inhiben la transferencia de un grupo fosfato desde el ATP al residuo tirosínico del receptor de tirosin quinasa. El mecanismo asociado con la cardiotoxicidad ${ }^{29}$, consiste en la generación de proteínas no plegadas que estimulan la respuesta de estrés del retículo endoplás- 


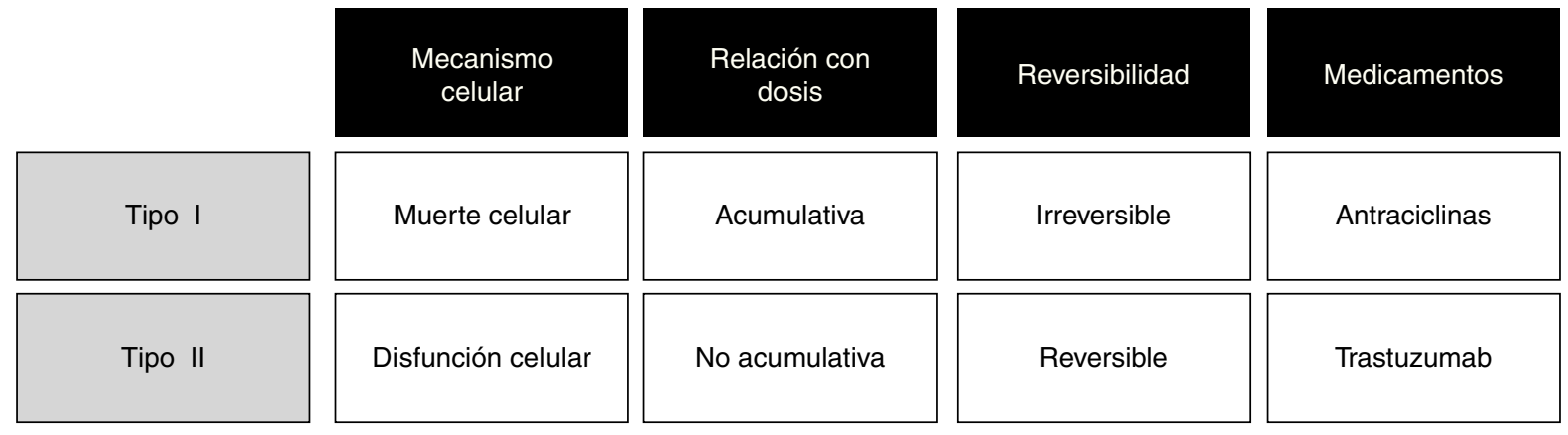

Figura 3 Tipos de medicamentos cardiotóxicos y mecanismo de acción.

mico (RE), activando las vías PERK e IRE1 (protein kinase $\mathrm{R}$-like endoplasmic reticulum kinase, inositol-requiring kinase 1) los cuales son receptores protein quinasa transmembrana residentes en el RE que censan señales agresoras provenientes de la acumulación de proteínas no plegadas, produciendo una autofosforilación con la subsecuente apoptosis por la activación de caspasas y proteínas homologas C/BEP (CHOP) $)^{21,30}$, (fig. 2).

\section{Otros agentes cardiotóxicos}

Dentro del arsenal terapéutico oncológico (fig. 1), la ciclofosfamida, un agente alquilante, que se caracteriza por su capacidad de daño al DNA y que se utiliza en el manejo de algunas leucemias y en trasplantes, induce la cardiotoxicidad aguda que se manifiesta desde las alteraciones electrocardiográficas sutiles hasta la cardiomiopatía que puede ser fatal; su efecto es dosis dependiente y es más común a dosis superiores a $200 \mathrm{mg} / \mathrm{kg}$. Sus efectos fatales se han reportado hasta en $11 \%$ de los casos. La miocarditis y menos común la falla cardiaca, aparecen durante las primeras semanas post tratamiento. Los derivados pirimidínicos como el 5- Fluoruracil (5-FU), actúan como antimetabolitos impidiendo la síntesis de DNA y RNA. Dentro de las manifestaciones de cardiotoxidad están: las arritmias, la isquemia, la falla cardiaca y la muerte súbita. Estas manifestaciones se presentan con síntomas de angina pectoris secundaria a un espasmo coronario y su incidencia es de $1-18 \%$. El profármaco del (5-FU) la capecitabina se ha asociado a la misma frecuencia de eventos cardiovasculares adversos. La interleuquina 2 (IL-2) se ha asociado con el desarrollo de la miocarditis y la cardiomiopatía en pacientes con carcinoma de células renales y melanoma; a su vez, se asocia a la presencia de: hipotensión, fibrilación atrial y taquicardia ventricular, debido a las alteraciones en la microcirculación. La toxicidad por paclitaxel se presenta en forma de: bradicardia, bloqueo cardiaco o arritmias atriales o ventriculares, secundario a un efecto cronotrópico directo en el sistema de Purkinje, presentando una incidencia de $0.5 \%$. El inhibidor del factor de crecimiento vascular endotelial (VEGF), el bevacizumab se asocia a: la disfunción miocárdica, la hipertensión y los eventos arteriales tromboembólicos con incidencia de $1.6 \%$, debido a una disminución en la producción del óxido nítrico. Los inhibidores multiquinasa, sutinib y sorafenib, al inhibir el receptor VEGF, se asocian a: la angina pectoris, la disminución en la fracción de eyección ventricular izquierda, la disfunción contráctil y las alteraciones
Alteraciones cardiovasculares de la quimioterapia

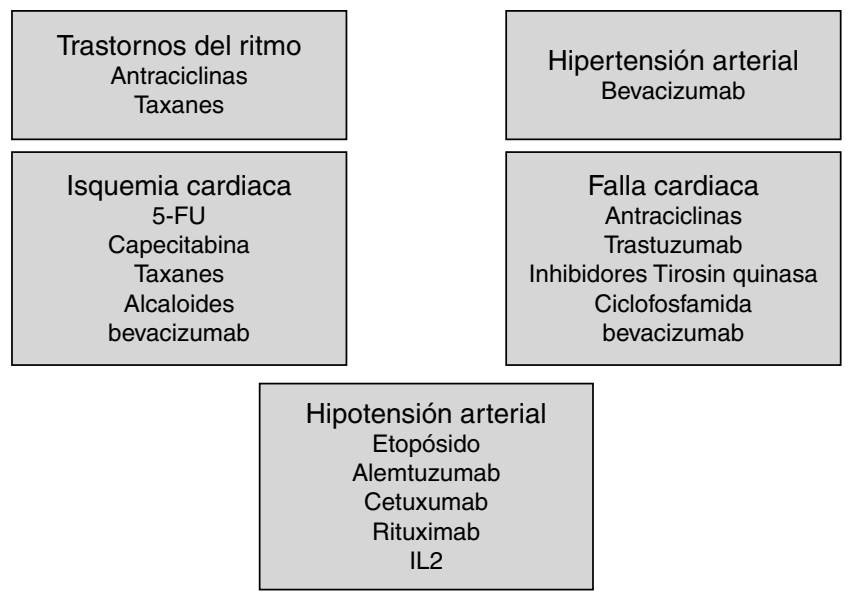

Figura 4 Medicamentos antineoplásicos con su respectivo trastorno cardiovascular. 5-FU 5- Fluorouracil, IL2 Interleuquina 2.

en ECG en 18, 12 y 16\%, respectivamente. Los interferones alfa 2 a y alfa 2 b indicados en neoplasias como: el melanoma, la leucemia mieloide crónica, el cáncer de células renales y el linfoma no Hodgkin, pueden causar arritmias que van desde una fibrilación atrial hasta una fibrilación ventricular en el $20 \%$ de los pacientes; y el uso crónico puede llevar a la cardiomiopatía dilatada ${ }^{14,21}$, (fig. 4).

\section{Monitoreo de la cardiotoxicidad}

El monitoreo de la cardiotoxicidad durante el tratamiento oncológico inicia con una evaluación general de las comorbilidades y los factores de riesgo cardiovasculares, una educación sobre estilos de vida saludable para el control de los factores de riesgo como: la hipertensión y la dislipidemia, y medidas para disminuir la cardiotoxicidad como el monitoreo durante la administración de medicamentos como: el 5-FU y el paclitaxel, para garantizar un monitoreo constante antes, durante y después de la terapia oncológica ${ }^{31}$.

La evaluación clínica basal y el uso del electrocardiograma es importante en pacientes que inician la terapia con: las antraciclinas, el paclitaxel y los inhibidores de la tirosin quinasa, ya que se convierte en una herramienta diagnóstico 
y pronóstico al detectar tempranamente signos de cardiomiopatía y alteraciones que favorezcan el inicio de arritmias como la prolongación del intervalo QT. El ecocardiograma con doppler debe ser empleado para evaluar la función cardiaca en pacientes sometidos a estas terapias; este método permite la evaluación de la función sistólica y diastólica con su fracción de eyección del ventrículo izquierdo (FEVI) y el acortamiento fraccional del ventrículo izquierdo, y a su vez, identificar alteraciones a nivel valvular y pericárdica ${ }^{31}$. La FEVI tiene unos parámetros para ser interpretados y su medición periódica se recomienda en pacientes que se encuentran usando las antraciclinas y los anticuerpos monoclonales, en especial con presencia de factores de riesgo, su evaluación depende de las dosis administradas y se recomienda una evaluación por cardiología o suspensión de la terapia de acuerdo a los hallazgos obtenidos:

\section{Antraciclinas}

1. Recomendaciones con $\mathrm{FEVI}>50 \%$ de la basal: - Medirla a los 250 a $300 \mathrm{mg} / \mathrm{m}^{2}$ de dosis acumulada.

- Medirla a los $450 \mathrm{mg} / \mathrm{m}^{2}$ de dosis acumulada.

- Medirla antes de cada dosis después de los $450 \mathrm{mg} / \mathrm{m}^{2}$ de dosis acumulada.

- Descontinuar la quimioterapia si hay una caída del $10 \%$ de la FEVI basal y la FEVI es < del 50\%.

2. Recomendaciones con $\mathrm{FEVI}<50 \%$ de la basal:

- No dar tratamiento si la FEVI es < del $30 \%$.

- Hacer mediciones seriadas antes de cada dosis.

- Descontinuar la quimioterapia si la FEVI cae $>10 \%$ de la basal o la FEVI es $<30 \%^{32}$.

\section{Trastuzumab}

Tener una $\mathrm{FEVI}<54 \%$ se asocia como factor de riesgo para desarrollar la falla cardiaca. Por lo cual, siempre al inicio de un régimen con este tipo de medicación se debe:

1. Tener una FEVI basal con ecografía $3 \mathrm{D}$ (idealmente) 0 ecografía 2D.

2. Evaluación del Strain Longitudinal Global (SLG) por ecocardiografía de rastreo de moteado (Speckle Tracking).

3. Medición de Troponina I.

Se debe remitir a cardiología si:

1. $\mathrm{FEVI}<53 \%$ (Considerar confirmación con RNM).

2. SLG menor de límite inferior normal.

3. Troponina positiva.

Se realiza seguimiento cada 3 meses durante la terapia si:

1. $\mathrm{FEVI}>53 \%$.

2. SLG mayor del límite inferior normal.

3. Troponina negativa ${ }^{32}$.

Con los estadios subclínicos, la ecocardiografía define la disfunción cardiaca relacionada a toxicidad a una caída del $10 \%$ a una $\mathrm{FEVI}<53 \%$, pero a pesar de ser el método de elección en la evaluación de los pacientes en quimioterapia, estos cambios pueden ser muy sutiles y a veces solo ser detectados en etapas tardías; por esto la detección temprana, incluso antes de desarrollar síntomas, se vuelve una prioridad. Para mejorar su desempeño, los índices diastólicos derivados del doppler representan un índice temprano de la disfunción ventricular izquierda, la evaluación del patrón de flujo diastólico mitral, la relación velocidad de flujo pico temprano a velocidad de flujo pico atrial $(E / A)$, el tiempo de desaceleración de la onda $E$ y la relajación isovolumétrica, son de gran utilidad para detectar alteraciones diastólicas del ventrículo izquierdo antes que una disfunción sistólica ocurra. A su vez, la ecocardiografía con rastreo de moteado (Speckle Tracking) permite una detección del daño del miocardio en fases tempranas, ya que es altamente sensible de la función cardiaca y provee información de la deformación miocárdica de forma objetiva. Mediante el SLG se pueden establecer parámetros de disfunción tempranos: cambios del valor basal $<8 \%$ no demuestra evidencia subclínica de disfunción ventricular, mientras que cambios $>15 \%$, representan una disfunción subclínica del ventrículo izquierdo. En caso de no contar con un valor basal, un valor absoluto con una caída $<19 \%$, es sugestivo de la cardiotoxicidad ${ }^{33}$. El doppler con pulsación tisular se puede realizar durante la evaluación ecocardiográfica regular y permite proveer información cuantitativa entorno a la relajación diastólica y el desempeño sistólico evaluando cambios tempranos en la función miocárdica del ventrículo izquierdo ${ }^{31}$.

\section{Técnicas imagenológicas adicionales}

1. Resonancia nuclear magnética: considerada como el método de elección para la evaluación de la masa cardiaca, los volúmenes y la fracción de eyección. Dada la ausencia de la utilización de presunciones geométricas le da la alta precisión. Utilizada principalmente cuando las imágenes de ecocardiografía son subóptimas o cuando hay discrepancia en la medición de la FEVI.

2. Técnicas de medicina nuclear: tiene alta reproducibilidad en el cálculo de la fracción de eyección con baja variabilidad interobservador. Como desventajas están: la exposición a radiación ionizante y la imposibilidad de valorar la función del ventrículo derecho, las válvulas y el pericardio.

La biopsia endomiocárdica representa el método más fidedigno para evaluar la lesión miocárdica, no obstante, al ser invasiva, obtener pequeñas porciones del miocardio, no aportar información funcional y al ser poco utilizada se ha limitado su uso en el monitoreo y el diagnóstico de la cardiotoxicidad $^{31}$.

El uso de los biomarcadores provee información importante acerca del mecanismo subyacente de la cardiotoxicidad. Las troponinas detectan: la isquemia celular en fases tempranas prediciendo morbilidad y mortalidad subclínica y clínica, su uso es limitado por la necesidad de controles seriados ${ }^{34}$. Los péptidos natriuréticos principalmente, el péptido natriurético cerebral (BNP), permiten una evaluación de estados de sobrecarga de volumen, representando presiones de llenado aumentadas, que ocurren en 
disfunción de ventrículo izquierdo; su valor puede aumentar cuando la falla cardiaca está en estado subclínico. Los valores elevados se correlacionan con disfunción ventricular izquierda en pacientes con terapia oncológica ${ }^{31}$.

\section{Prevención y manejo de la cardiotoxicidad inducida por QT}

Para prevenir el daño miocárdico sin disminuir la eficacia terapéutica en el tratamiento oncológico, se deben tener en cuenta: los factores independientes de riesgo para desarrollar la cardiotoxicidad. La dosis acumulada de antraciclinas, la raza africano americano, las edades extremas, la diabetes, la hipertensión, el sobrepeso o el bajo peso y las comorbilidades severas; indican un riesgo para desarrollar eventos cardiovasculares ${ }^{35}$, de esta manera se pueden aplicar medidas preventivas como:

1. Cambios en los esquemas terapéuticos prefiriendo la aplicación en infusión prolongadas a los bolos, con esquemas que van desde 6 hasta 96 horas, ya que hay un riesgo 4.13 veces mayor de desarrollar la cardiotoxicidad con la aplicación en bolos ${ }^{36}$.

2. El uso de las antraciclinas con recubrimiento liposomal, las cuales evitan la entrada al miocardio sin afectar la penetrancia tumoral, con una disminución de la cardiotoxicidad hasta en un $80 \%$ comparada con formas convencionales ${ }^{37}$.

3. El uso de análogos de las antraciclinas como la epirrucbicina y la mitoxantrona, que a pesar de su disminución en la eficacia terapéutica, presentan una menor cardiotoxicidad.

4. La utilización del agente quelante de hierro, el dexrazoxane, el cual inhibe la peroxidación de las membranas lipídicas con disminución de la cardiotoxicidad en las antraciclinas. Se usa al mismo tiempo que estos medicamentos o la primera dosis al inicio y la segunda dosis al llegar a dosis acumulada de $300 \mathrm{mg} / \mathrm{m}^{2}$.

5. El uso individual de cada agente cardiotóxico y la disminución de tiempos terapéuticos de medicamentos como el Trastuzumab ${ }^{20,21}$.

6. El estudio OVERCOME recomienda el uso de agentes inhibidores del eje neurohormonal como: los Inhibidores de la enzima convertidora de angiotensina (IECAS) y el carvedilol, los cuales logran una protección sobre los efectos cardiotóxicos al ser usados durante la terapia oncológica, mediante efectos antioxidantes ${ }^{38,39}$.

Cuando a pesar de usar terapia preventiva, la cardiotoxicidad se detecta bajo los parámetros establecidos ya revisados por la ecocardiografía u otros métodos diagnósticos, se debe suspender el medicamento agresor e instaurar un tratamiento adecuado, aun en aquellos pacientes que asintomáticos tienen disfunción ventricular izquierda.

La sugerencia del tratamiento se basa en los IECAS y los betabloqueadores; al presentarse síntomas y estados francos de la falla cardiaca, se debe instaurar un tratamiento adecuado que integra: los diuréticos, los antagonistas de la aldosterona, los nitratos y aunque controvertido, la digoxina en casos muy sintomáticos o en presencia de trastornos del ritmo. De acuerdo a la evolución y respuesta clínica se planteará la pertinencia de reiniciar o cambiar régimen quimioterapéutico ${ }^{5,21}$.

\section{Conclusiones}

La cardiotoxicidad inducida por quimioterapia, en especial con agentes como las antraciclinas y el trastuzumab, representa un riesgo para los pacientes en la terapia oncológica. No obstante, el entendimiento de los mecanismos fisiopatológicos de la enfermedad ha llevado a plantear medidas de: la prevención, el diagnóstico y el tratamiento, que han permitido una reducción en la incidencia con mejoría en la sobrevida de estos pacientes. La necesidad de una detección temprana que permita iniciar terapias precoces y preventivas en algunos casos, requiere de estudios utilizando los diferentes métodos diagnósticos o modalidades adicionales a la ecocardiografía convencional, para lograr aumentar la sensibilidad y especificidad, logrando un impacto en etapas más tempranas. De esta manera, la cardio-oncología se posiciona como una especialidad que puede permitir una mejoría en el tratamiento y calidad de vida de los pacientes sometidos a estas terapias, y se establece como un área de interés para futuras investigaciones.

\section{Responsabilidades éticas}

Protección de personas y animales. Los autores declaran que para esta investigación no se han realizado experimentos en seres humanos ni en animales.

Confidencialidad de los datos. Los autores declaran que en este artículo no aparecen datos de pacientes.

Derecho a la privacidad y consentimiento informado. Los autores declaran que en este artículo no aparecen datos de pacientes.

\section{Conflicto de intereses}

Los autores declaran no tener ningún conflicto de intereses.

\section{Agradecimientos}

Al doctor Juan Guillermo Echeverri. Médico Internista, Cardiólogo, Ecocardiografista. Instituto CEMDE SAS Medellín, Colombia; por su aporte en la sección de métodos diagnósticos.

\section{Bibliografía}

1. Siegel RL, Miller KD, Jemal A. Cancer statistics, 2015: Cancer Statistics, 2015. CA Cancer J Clin. 2015 Jan;65(1):5-29.

2. DeSantis CE, Lin CC, Mariotto AB, Siegel RL, Stein KD, Kramer JL, et al. Cancer treatment and survivorship statistics, 2014: Cancer Treatment and Survivorship Statistics, 2014. CA Cancer J Clin. 2014 Jul;64(4):252-71.

3. Yusuf SW, Sami S, Daher IN. Radiation-induced heart disease: a clinical update. Cardiol Res Pract. 2011;2011:317659.

4. Carver JR, Shapiro CL, Ng A, Jacobs L, Schwartz C, Virgo KS, et al. American Society of Clinical Oncology clinical evidence review on the ongoing care of adult cancer survivors: cardiac 
and pulmonary late effects. J Clin Oncol Off J Am Soc Clin Oncol. 2007 Sep 1;25(25):3991-4008.

5. Florescu M, Cinteza M, Vinereanu D. Chemotherapy-induced Cardiotoxicity. Mædica. 2013;8(1):59-67.

6. Seidman A, Hudis C, Pierri MK, Shak S, Paton V, Ashby $M$, et al. Cardiac dysfunction in the trastuzumab clinical trials experience. J Clin Oncol Off J Am Soc Clin Oncol. 2002;20(5):1215-21. Mar 1.

7. Jiji RS, Kramer CM, Salerno M. Non-invasive imaging and monitoring cardiotoxicity of cancer therapeutic drugs. J Nucl Cardiol Off Publ Am Soc Nucl Cardiol. 2012;19(2):377-88.

8. Albini A, Pennesi G, Donatelli F, Cammarota R, De Flora S, Noonan DM. Cardiotoxicity of anticancer drugs: the need for cardio-oncology and cardio-oncological prevention. J Natl Cancer Inst. 2010;102(1):14-25. Jan 6.

9. Dolci A, Dominici R, Cardinale D, Sandri MT, Panteghini M. Biochemical markers for prediction of chemotherapy-induced cardiotoxicity: systematic review of the literature and recommendations for use. Am J Clin Pathol. 2008;130(5):688-95.

10. Frishman WH, Sung HM, Yee HC, Liu LL, Keefe D, Einzig Al, et al. Cardiovascular toxicity with cancer chemotherapy. Curr Probl Cancer. 1997;21(6):301-60.

11. Pai VB, Nahata MC. Cardiotoxicity of chemotherapeutic agents: incidence, treatment and prevention. Drug Saf. 2000;22(4):263-302.

12. Rang HP. Rang et Dale's pharmacology. Edinburgh [u.a.]: Elsevier, Churchill Livingstone; 2016.

13. Figueredo VM, Chemical Cardiomyopathies. The Negative Effects of Medications and Nonprescribed Drugs on the Heart. Am J Med. 2011 Jun;124(6):480-8.

14. Zylberman M, Agüero R. Consenso de diagnóstico, prevención y tratamiento de la cardiotoxicidad por tratamiento médico del cáncer. Rev Argent Cardiol. 2013;81 suplemento 5:1-64.

15. Brana I, Tabernero J. Cardiotoxicity. Ann Oncol. 2010;21 Supplement 7:vii173-9. Oct 1.

16. Lefrak EA, Pitha J, Rosenheim S, Gottlieb JA. A clinicopathologic analysis of adriamycin cardiotoxicity. Cancer. 1973;32(2):302-14.

17. Ewer MS, Ali MK, Mackay B, Wallace S, Valdivieso M, Legha SS, et al. A comparison of cardiac biopsy grades and ejection fraction estimations in patients receiving Adriamycin. J Clin Oncol Off J Am Soc Clin Oncol. 1984 Feb;2(2):112-7.

18. Klaassen CD, Casarett LJ, Doull J, editores. Casarett and Doull's toxicology: the basic science of poisons. 8 th ed. New York: McGraw-Hill Education; 2013. p. 1454, p.

19. Khakoo AY, Liu PP, Force T, Lopez-Berestein G, Jones LW, Schneider J, et al. Cardiotoxicity due to cancer therapy. Tex Heart Inst J Tex Heart Inst St Lukes Episcop Hosp Tex Child Hosp. 2011;38(3):253-6.

20. Schlitt A, Jordan K, Vordermark D, Schwamborn J, Langer T, Thomssen C. Cardiotoxicity and oncological treatments. Dtsch Ärztebl Int. 2014;111(10):161-8. Mar 7.

21. Hurtado S, Mejía AM, Sanabria A. Cardiotoxicidad por quimioterapia Un enfoque práctico para el clínico. Insufic Card. 2011;6(3):131-43.

22. Adão R, de Keulenaer G, Leite-Moreira A, Brás-Silva C. Cardiotoxicidade associada à terapêutica oncológica: mecanismos fisiopatológicos e estratégias de prevenção. Rev Port Cardiol. 2013;32(5):395-409.

23. Slørdal L, Spigset O. Heart failure induced by non-cardiac drugs. Drug Saf. 2006;29(7):567-86.
24. Curigliano G, Cardinale D, Suter T, Plataniotis G, de Azambuja E, Sandri MT, et al. Cardiovascular toxicity induced by chemotherapy, targeted agents and radiotherapy: ESMO Clinical Practice Guidelines. Ann Oncol. 2012 Oct 1;23 suppl 7:vii155-66.

25. Ozcelik C, Erdmann B, Pilz B, Wettschureck N, Britsch S, Hubner $\mathrm{N}$, et al. Conditional mutation of the ErbB2 (HER2) receptor in cardiomyocytes leads to dilated cardiomyopathy. Proc Natl Acad Sci. 2002;99(13):8880-5. Jun 25.

26. Serrano C, Cortés J, De Mattos-Arruda L, Bellet M, Gómez P, Saura C, et al. Trastuzumab-related cardiotoxicity in the elderly: a role for cardiovascular risk factors. Ann Oncol Off J Eur Soc Med Oncol ESMO. 2012;23(4):897-902.

27. Di Cosimo S. Heart to heart with trastuzumab: a review on cardiac toxicity. Target Oncol. 2011;6(4):189-95.

28. Chavez-MacGregor M, Zhang N, Buchholz TA, Zhang Y, Niu J, Elting L, et al. Trastuzumab-Related Cardiotoxicity Among Older Patients With Breast Cancer. J Clin Oncol. 2013;31(33):4222-8. Nov 20.

29. Orphanos GS, Ioannidis GN, Ardavanis AG. Cardiotoxicity induced by tyrosine kinase inhibitors. Acta Oncol. 2009;48(7):964-70.

30. Liu Z, Lv Y, Zhao N, Guan G, Wang J. Protein kinase R-like ER kinase and its role in endoplasmic reticulum stress-decided cell fate. Cell Death Dis. 2015;6(7):e1822. Jul 30.

31. Bovelli D, Plataniotis G, Roila F, On behalf of the ESMO Guidelines Working Group. Cardiotoxicity of chemotherapeutic agents and radiotherapy-related heart disease: ESMO Clinical Practice Guidelines. Ann Oncol. 2010;21 Supplement 5:v277-82. May 1.

32. Plana JC, Galderisi M, Barac A, Ewer MS, Ky B, Scherrer-Crosbie $M$, et al. Expert Consensus for Multimodality Imaging Evaluation of Adult Patients during and after Cancer Therapy: A Report from the American Society of Echocardiography and the European Association of Cardiovascular Imaging. J Am Soc Echocardiogr. 2014;27(9):911-39.

33. Dogru A, Cabuk D, Sahin T, Dolasik I, Temiz S, Uygun K. Evaluation of Cardiotoxicity via Speckle-Tracking Echocardiography in Patients Treated with Anthracyclines. Oncol Res Treat. 2013;36(12), 1-1.

34. Reagan WJ, York M, Berridge B, Schultze E, Walker D, Pettit S. Comparison of Cardiac Troponin I and T, Including the Evaluation of an Ultrasensitive Assay, as Indicators of Doxorubicin-induced Cardiotoxicity. Toxicol Pathol. 2013;41(8):1146-58. Dec 1.

35. Lotrionte M, Biondi-Zoccai G, Abbate A, Lanzetta G, D’Ascenzo F, Malavasi V, et al. Review and Meta-Analysis of Incidence and Clinical Predictors of Anthracycline Cardiotoxicity. Am J Cardiol. 2013;112(12):1980-4.

36. Smith LA, Cornelius VR, Plummer CJ, Levitt G, Verrill M, Canney $\mathrm{P}$, et al. Cardiotoxicity of anthracycline agents for the treatment of cancer: systematic review and meta-analysis of randomised controlled trials. BMC Cancer. 2010;10:337.

37. Rahman AM, Yusuf SW, Ewer MS. Anthracycline-induced cardiotoxicity and the cardiac-sparing effect of liposomal formulation. Int J Nanomedicine. 2007;2(4):567-83.

38. Bosch X, Rovira $M$, Sitges $M$, Domènech A, Ortiz-Pérez JT, de Caralt TM, et al. Enalapril and Carvedilol for Preventing Chemotherapy-Induced Left Ventricular Systolic Dysfunction in Patients With Malignant Hemopathies. J Am Coll Cardiol. 2013;61(23):2355-62.

39. Smiseth OA, Edvardsen T, Skulstad H. Cardioprotection During Chemotherapy. J Am Coll Cardiol. 2013;61(23):2363-4. 